\title{
Guided Bloch Surface Waves on Ultrathin Polymeric Ridges
}

\section{Emiliano Descrovi, ${ }^{*}{ }^{\dagger}$ Tristan Sfez, ${ }^{\mp}$ Marzia Quaglio, ${ }^{\S}$ Daniele Brunazzo," Lorenzo Dominici, ${ }^{\perp}$ Francesco Michelotti, ${ }^{\perp}$ Hans Peter Herzig, ${ }^{\ddagger}$ Olivier J. F. Martin, ${ }^{\prime \prime}$ and Fabrizio Giorgis ${ }^{\dagger}$}

\author{
${ }^{\dagger}$ Materials and Microsystems Laboratory $\chi$ Lab, Politecnico di Torino, C.so Duca degli Abruzzi 24, IT-10129 Torino, Italy, \\ † Optics and Photonics Technology Laboratory, Ecole Polytechnique Fédérale de Lausanne EPFL-STI-OPT, A.-L. \\ Breguet 2, CH-2000 Neuchâtel, Switzerland, ${ }^{\S}$ IIT - Italian Institute of Technology @ POLITO, Corso Trento 21, \\ 10129 Torino, Italy, "Nanophotonics and Metrology Laboratory, Swiss Federal Institute of Technology Lausanne \\ EPFL-STI-NAM, ELG Station 11, CH-1015 Lausanne, Switzerland, and ${ }^{\perp}$ Dipartimento di Energetica, SAPIENZA \\ Università di Roma and CNISM, Via A. Scarpa 16, IT-00161 Roma, Italy
}

\begin{abstract}
We present a direct evidence of Bloch surface waves (BSWs) waveguiding on ultrathin polymeric ridges, supported by near-field measurements. It is demonstrated that near-infrared BSWs sustained by a silicon-based multilayer can be locally coupled and guided through dielectric ridges of nanometric thickness with low propagation losses. Using a conventional prism-based configuration, we demonstrate a wavelength-selective BSW coupling inside and outside the ridge. Such a result can open interesting opportunities in surface wave-mediated sensing applications, where light could be selectively coupled in specific regions defined by nanometric reliefs
\end{abstract}

KEYWORDS Surface electromagnetic waves, near-field optical microscopy

B loch surface waves (BSW) are either TE- or TMpolarized surface modes that can be sustained by truncated stacks of periodically arranged dielectric layers. ${ }^{1,2}$ Although BSWs have been known for more than thirty years, they have been recently reconsidered as an alternative to surface plasmon polaritons (SPP), ${ }^{3}$ in particular in sensing applications. ${ }^{4-6}$ Until now, a number of far-field and near-field investigations have been conducted on the coupling of BSW on flat ${ }^{7-9}$ and corrugated planar structures. ${ }^{10-12}$ Here we demonstrate that near-infrared BSWs can be selectively prism-coupled and efficiently guided through an ultrathin polymeric ridge waveguide having thickness $<\lambda / 10$ realized onto a silicon nitride multilayer. Besides being wavelength scalable and fully compatible with the actual fabrication technologies of integrated photonic and plasmonic structures, the proposed hybrid organic/inorganic structure can provide disruptive opportunities in waveguide-based biosensing schemes, (see, e.g., ref 13) in which the chemical specificity of the sensor might be implemented by functional molecule layers patterned as waveguides with nanometric thickness.

In the past decade, a large number of issues connected to the guiding of electromagnetic surface waves (mostly SPP) on a subwavelength scale have been addressed by one branch of plasmonics. ${ }^{14}$ Plasmonic waveguides according to different geometries have been proposed mainly for

* To whom correspondence should be addressed: E-mail: emiliano.descrovi@ polito.it.

Received for review: 02/9/2010

Published on Web: 05/06/2010 gaining strong (lateral) field confinement while maintaining low propagation losses in view of a high-density packing of integrated photonic circuitry. ${ }^{15}$ In addition to guiding mechanisms based on ultrathin metallic membranes, ${ }^{16}$ nanoparticles chains, ${ }^{17,18}$ nanowires on dielectric substrates, ${ }^{19}$ dielectric nanocylinders on metallic films, ${ }^{20}$ or V-grooves in metal surfaces, ${ }^{21}$ the most popular plasmonic waveguide configurations are constituted by metal-insulator-metal $(\mathrm{MIM})^{22,23}$ or insulator-metal-insulator (IMI) ${ }^{24}$ structures. Among the latter, we recall the so-called dielectric-loaded surface plasmon polariton waveguides (DLSPPW), ${ }^{25}$ in which a dielectric stripe is deposited onto a flat metallic film. The dielectric cladding is thick enough to confine SPP within the ridge, therefore lowering the propagation losses as well as the sensitivity upon external perturbations. The latter feature makes DLSPPW not particularly well suited for sensing applications despite the millimeter range propagation constants that can be attained. Indeed, biochemical sensing can be provided in waveguiding arrangements where the field is confined in low-refractive index regions like water or air (e.g., slot waveguides ${ }^{26}$ ).

Here we demonstrate that an organic stripe having nanometric thickness can guide BSWs, while preserving the field confined on the top surface. We use an ultrathin polymeric ridge (refractive index $n_{\mathrm{wg}}=1.625$ ) on a properly designed multilayer sustaining TE-polarized BSW in the near infrared. In the case biosensing issues would be concerned, the polymer might be replaced by any biochemically functional material suitable for molecular recognition. At the cost of a lateral confinement in the range of few micrometers, guidance is experimentally evidenced for ridge thicknesses 


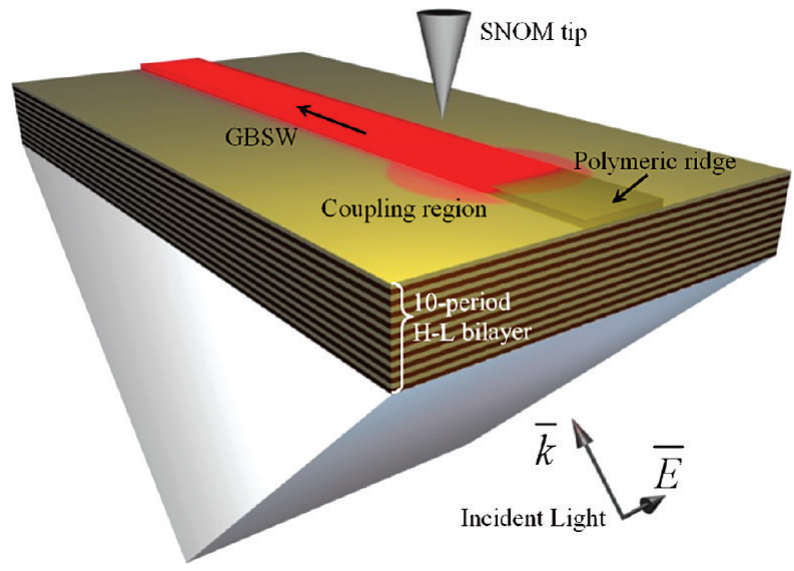

FIGURE 1. A slightly focused linearly polarized beam illuminates the multilayer and the waeguide ridge according to the Kretschmann configuration. Once the TE-polarization is set, a selective coupling of BSW inside or outside the ridge can be obtained depending on the radiation wavelength and the angle of incidence. The fibered SNOM tip raster scans the guide and the multilayer top surface.

down to approximately $30 \mathrm{~nm}$ with the actual low losses being mainly associated to the leakage through the substrate. Rigorous computational analysis, based on finite element method (FEM), suggests that even thinner organic ridges might be employed, while still preserving BSW guidance.

We consider an hydrogenated amorphous silicon nitride multilayer made of a 10 period stack of high $\left(n_{\mathrm{H}}=2.23\right.$ at $\lambda$ $=1530 \mathrm{~nm})$ and low $\left(n_{\mathrm{L}}=1.75\right.$ at $\left.\lambda=1530 \mathrm{~nm}\right)$ refractive index layers with thicknesses $d_{\mathrm{H}}=240 \mathrm{~nm}$ and $d_{\mathrm{L}}=294$ $\mathrm{nm}$ respectively, on a glass substrate. $\mathrm{a}-\mathrm{Si}_{1-x} \mathrm{~N}_{x}: \mathrm{H}$ is grown by plasma enhanced chemical vapor deposition (PECVD) on a Corning glass substrate. Depending on the nitrogen content, a-Si ${ }_{1-x} \mathrm{~N}_{x}: \mathrm{H}$ can have tunable refractive index and optical gaps over a wide energy range. The composition of the $\mathrm{a}-\mathrm{Si}_{1-x} \mathrm{~N}_{x}: \mathrm{H}$ layers was controlled by operating on the ammonia fraction present in a $\mathrm{SiH}_{4}+\mathrm{NH}_{3}$ plasma. ${ }^{9}$ A thin polymeric ridge waveguide, $3.5 \mu \mathrm{m}$ wide and $\sim 110 \mathrm{~nm}$ thick, is fabricated on the top surface of the multilayer by means of standard photolithographic techniques after spinning of a $\sim 110 \mathrm{~nm}$ thick positive photoresist AZ5214E (by Clariant $\mathrm{GmbH}$ ).

TE-polarized guided BSWs (GBSWs) are coupled according to the Kretschmann configuration ${ }^{27}$ after oil-contacting the substrate to the prism (refractive index $n_{\mathrm{p}}=1.5$ ). The top surface of the multilayer and the waveguide are raster scanned by a fibered scanning near-field optical microscope (SNOM) probe, as depicted in the sketch of Figure 1. The SNOM apparatus implements a multiheterodyne interferometric detection technique, able to independently detect complex optical fields after a simultaneous illumination of the sample by TE- and TM-polarized light. ${ }^{9}$ The multiheterodyne SNOM (MH-SNOM) is based on a classical SNOM in which a sharp optical fiber collects the electromagnetic field. In heterodyne SNOM, the scanning probe represents one branch of a Mach-Zender interferometer. A small frequency difference generated between the reference and object arms

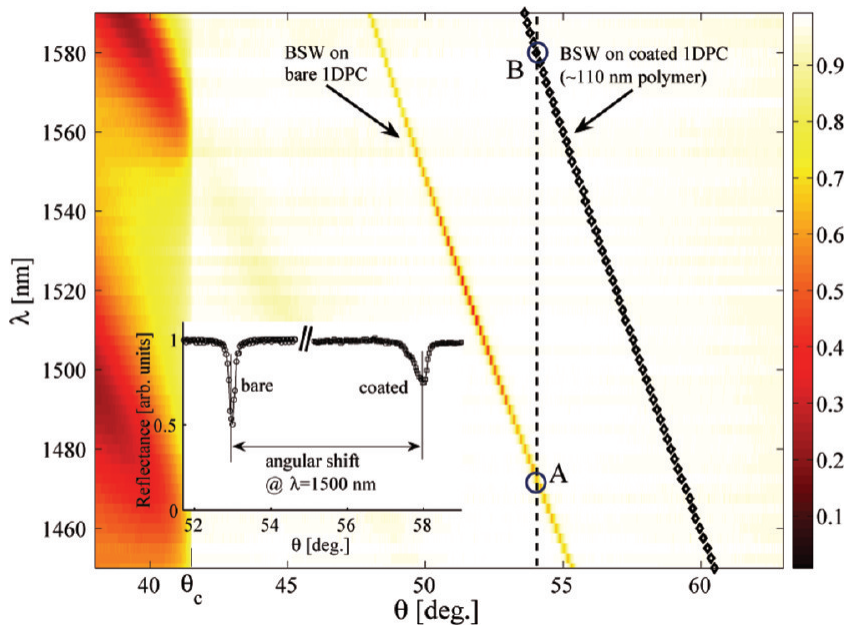

FIGURE 2. False color map of the reflectance $R(\theta, \lambda)$ measured by illuminating the bare multilayer at incident angles $\theta$ and wavelengths $\lambda$ in the Kretschmann configuration. Diamonds indicate the shifted position of BSW in the case where the bare multilayer surface is coated with a $110 \mathrm{~nm}$ thick polymeric film. (Inset) Reflectance dips (at $\lambda=1500 \mathrm{~nm}$ ) associated to BSW coupling on bare and coated multilayers.

provides a beat signal that allows the detection of both the amplitude and phase of the optical field collected by the probe. According to this scheme, the reference and object arms of the MH-SNOM are split again into two orthogonal states of polarization, each of the four resulting beams being differently shifted in frequency. The detected intensity is therefore a superposition of six beat signals, each one being independently demodulated. This original setup allows simultaneous detection of the amplitude and phase of the optical near-fields resulting from two orthogonal electric field components of the illuminating beam. The sample illumination is provided by a slightly focused fibered tunable laser diode emitting in the range [1460-1580] nm.

Before considering GBSW on the ridge waveguide, we provide an experimental evidence of BSWs on the planar multilayer. ${ }^{28}$ We determined a reflectance map $R(\theta, \lambda)$ of the multilayer, according to the Kretschmann configuration in a $\theta-2 \theta$ goniometric setup (Figure 2). Reflectance values mapped in false colors refer to the case of the bare multilayer. A spectrally narrow low-reflectance region running beyond the critical angle $\left(\theta_{\mathrm{C}}\right)$ represents the BSW dispersion curve, as indicated. BSW coupling occurs for those value pairs $\left(\theta_{\mathrm{BSW}}, \lambda_{\mathrm{BSW}}\right)$ identifying the angular and spectral position of reflectance dips. The propagation constants of BSWs can be calculated as $\beta_{\mathrm{BSW}}=2 \pi n_{\text {eff }} / \lambda_{\mathrm{BSW}}$ where the effective refractive index $n_{\text {eff }}=n_{\mathrm{p}} \sin \left(\theta_{\mathrm{BSw}}\right)$. Once the multilayer surface is coated with a thin polymeric film, the BSW propagation constants shift to higher values, depending on the polymer refractive index and thickness. In Figure 2, the shifted BSW dispersion curve (diamonds), measured after spin-coating a polymeric film $\sim 110 \mathrm{~nm}$ thick is also indicated. Resonance dips associated to BSW on the bare and the coated multilayer are spectrally and angularly well 


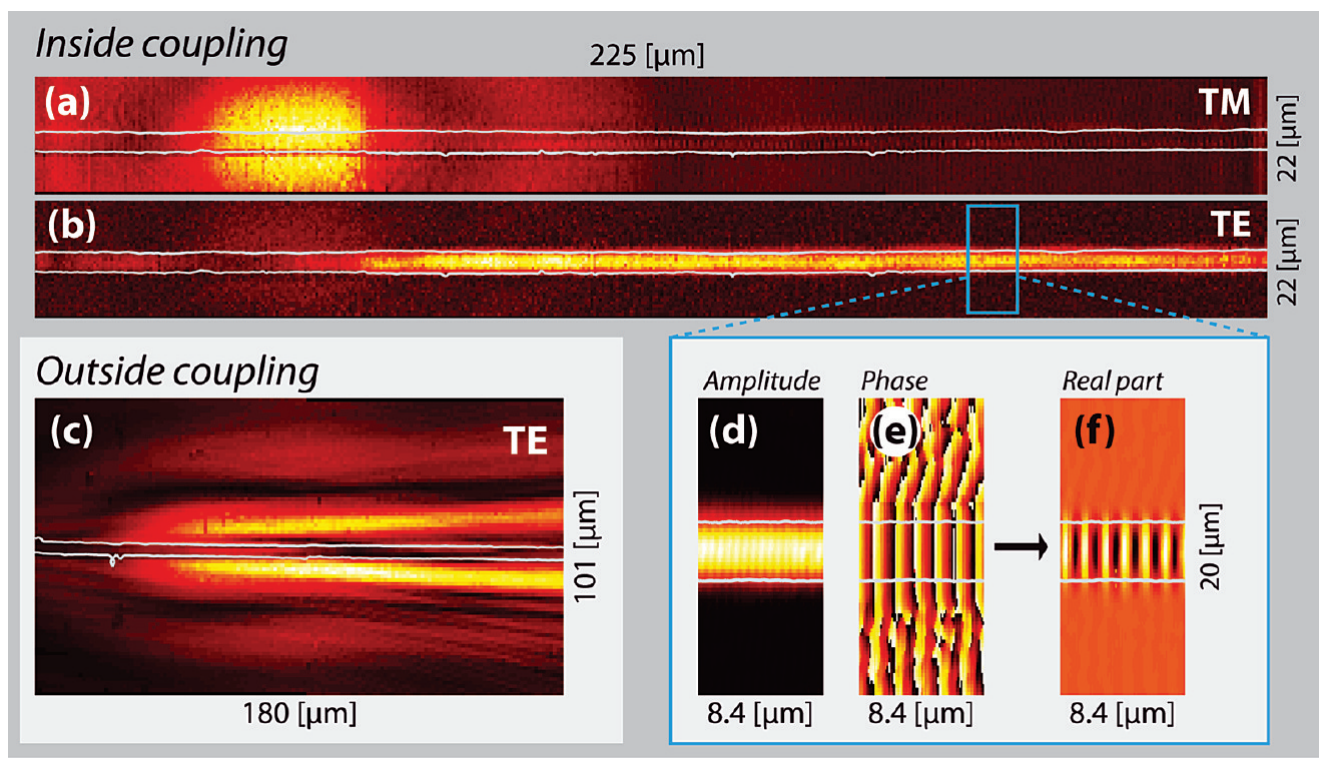

FIGURE 3. (a) Airy-like near-field amplitude distribution upon TM-polarized illumination at $\lambda_{\mathrm{BSW}}=1580 \mathrm{~nm}$. (b) Guided BSW field amplitude distribution on the polymeric ridge upon TE-polarized illumination at $\lambda_{\mathrm{BSW}}=1580 \mathrm{~nm}$. (c) BSW field amplitude distribution on the a-Si ${ }_{1-x} \mathrm{~N}_{x}: \mathrm{H}$ surface outside the polymeric ridge after TE-polarized illumination at $\lambda_{\mathrm{BSW}}=1470 \mathrm{~nm}$. The angle of incidence of the incident beam is kept fixed. Zoom: (e) amplitude, (f) phase, and (g) real-part field distributions of GBSW far away from the coupling region. The boundaries of the ridge waveguide as measured by the SNOM shear-force are indicated by the bright contourplot.

separated (see inset). Such a separation, together with the remarkable narrowness of BSW dips, provides the basic mechanism for obtaining a good confinement of GBSW in the polymeric ridge.

Turning now to the case of the waveguide, if the ridge is prism-illuminated roughly according to the dispersion curve of BSW on the coated multilayer, it is evidenced that guidance of BSW can be obtained. This is shown in Figure 3a,b, where the TM- and TE-polarized fields amplitude obtained with an illumination angle $\theta_{\mathrm{BSW}} \simeq 54.02^{\circ}$ and wavelength $\lambda_{\mathrm{BSW}}=1580 \mathrm{~nm}$ (point $\mathrm{B}$ in Figure 2) are respectively presented as measured by the SNOM.

Specifically, Figure 3 a shows the field amplitude distribution on the multilayer surface, when a TM-polarized focused beam is used for illumination. The evanescent field is mainly distributed according to the projection of an Airy-like pattern, as already evidenced elsewhere. ${ }^{9}$ More interestingly, Figure 3b shows that a TE-polarized GBSW is actually guided along the ridge, showing a transversal distribution with a welldefined central lobe and a very slow amplitude decrease in the propagation direction.

If the illumination wavelength is varied to $\lambda_{\mathrm{BSW}}=1470$ $\mathrm{nm}$, while the illumination angle is kept fixed (point $\mathrm{A}$ in Figure 2), the field distribution shown in Figure $3 \mathrm{c}$ is detected, in which BSW is excited only outside the ridge. As a result, the typical cometlike pattern ${ }^{9}$ is shadowed by the ridge itself. This effect is expected from the dispersion curves shown in Figure 2, showing that BSWs cannot be coupled on the ridge at such a wavelength and incidence angle.

A highly resolved scan over $8.4 \mu \mathrm{m} \times 20 \mu \mathrm{m}$ allows us to appreciate a closer view of the spatial distribution of the GBSW complex field. In Figure 3d-f, we show the measured amplitude, phase, and the resulting real part of GBSW field far away from the region of direct illumination. Wavefronts are flat and well confined within the ridge boundaries, as expected for the fundamental mode on a rectangular waveguide. $^{29}$ The field amplitude appears only slightly attenuated in the propagation direction due to the leakage through the substrate. After performing a Fourier analysis on SNOM measurements over a wider scan area, we found the spatial modulation frequency of this fundamental mode is $f \simeq 0.736 \pm 0.01 \mu \mathrm{m}^{-1}$, which is slightly smaller than the estimation from the measured dispersion curve in Figure 2, that is, $n_{\mathrm{p}} \sin \left(\theta_{\mathrm{BSW}}\right) / \lambda=0.768 \mu \mathrm{m}^{-1}$, as expected. Substantial deviations of the GBSW dispersion from the BSW dispersion curve on the flat, coated multilayer are expected especially if higher order modes are considered or the ridge width is sensibly reduced. ${ }^{28}$

A computational FEM model of the overall structure can be built, including a semi-infinite glass substrate, the truncated multilayer and the polymeric ridge. Since BSWs excited by the Kretschmann configuration are coupled back to the substrate and leak out through the prism, in principle they could not be considered as pure modes of the structure. However, owing to the fact that the coupling is weak, they can be treated as leaky modes with real and imaginary parts of the effective index, as calculated with FEM, the latter bearing the information on leakage. The spatial distribution and effective refractive index of GBSWs are calculated using the commercial finite-element package COMSOL Multyphysics v.3.5a. The mode analyzer of the RF module was used to find the fundamental mode of several waveguides profiles. The calculation region was enclosed in perfectly matched layers (PML) to avoid reflections at the boundaries. 


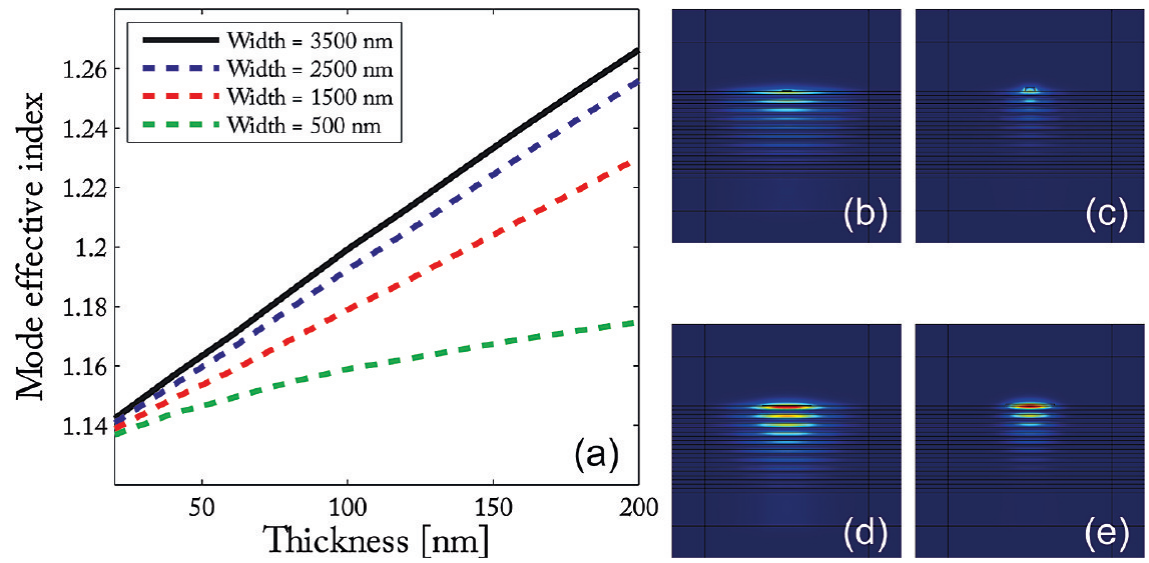

FIGURE 4. (a) Effective refractive index of the fundamental GBSW mode for different geometrical parameters (width $w$ and thickness $t$ ) of the rectangular ridge. $(b-e)$ GBSW total intensity distributions showing different degree of lateral confinement according to the effective refractive index dispersion curves.

Results showing the dependence of the real part of the complex effective index $n_{\text {eff }}$ of the GBSW with respect to the waveguide geometrical parameters are presented in Figure 4a.

Ridges with varying thickness $(t)$ and width $(w)$ provide different degree of lateral confinement of GBSW (Figure $4 b-e)$, while the vertical field confinement at the top surface is still assured for small ridge thicknesses. For relatively large ridge width $(>2 \mu \mathrm{m})$, a weak dependence of $n_{\mathrm{eff}}$ over the ridge width is observed, as in low index contrast waveguides. On the other hand, the strong increase of $n_{\mathrm{eff}}$ as the ridge thickness increases indicates that the mode is more and more confined within the polymeric region, until a conventional rectangular waveguide guided mode is obtained. For tiny ridges ( $w=600 \mathrm{~nm}, t=20 \mathrm{~nm}$ ), the lateral confinement is rather weak (Figure $4 \mathrm{~b}$ ). Similarly to DLSPPW, some improvements on the lateral confinement can be obtained as the ridge thickness is increased, as in the case of the $w=600 \mathrm{~nm}, t=200 \mathrm{~nm}$ ridge in Figure 4c, where the field appears almost completely confined in the polymer. Wider ridges (in the micrometer scale) allow to maintain a strong binding of GBSW on the surface even in the case of very small thickness (see the case of a $w=3 \mu \mathrm{m}, t=20 \mathrm{~nm}$ ridge shown in Figure 4d). In Figure 4e, the case of the $w=$ $3.5 \mu \mathrm{m}, t=110 \mathrm{~nm}$ ridge investigated experimentally is presented.

The selectivity of GBSW coupling has been experimentally investigated on an ultrathin Teflon ridge $30 \mathrm{~nm}$ thick. To ease the fabrication of a extremely thin polymeric film with an acceptable homogeneity, a RIE process involving Teflon deposition has been employed instead of the more conventional polymer spinning. Results are shown in Figure $5 a, b$, where SNOM mappings are shown corresponding to the coupling inside and outside the waveguide respectively. The advantage of this configuration is to provide a micrometric lateral confinement while preserving the typical surface mode features required for sensing applications.

In conclusion, we demonstrated the possibility of guiding BSW by patterning a suitable multilayer with dielectric stripe

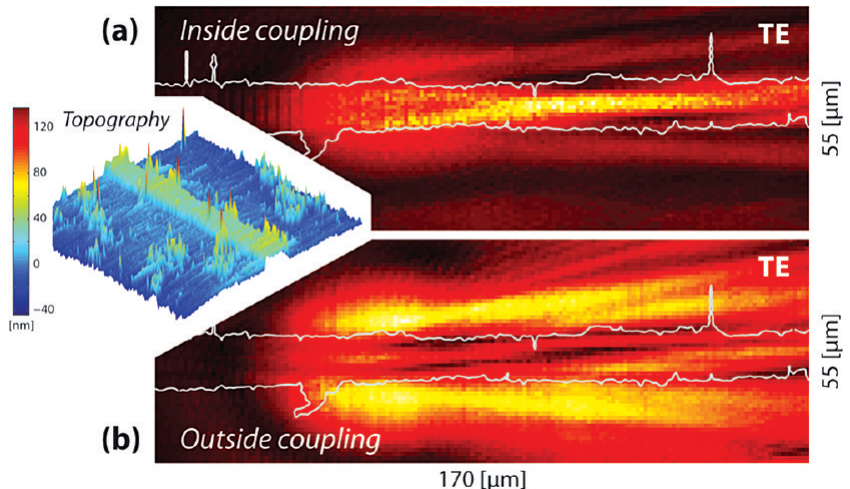

FIGURE 5. Wavelength-selective GBSW coupling (a) inside and (b) outside a $30 \mathrm{~nm}$ thick Teflon ridge as measured by the SNOM. Inset: a 3D surface representation of the Teflon ridge topography as shearforce measured.

having nanometric thickness. Contrary to DLSPPWs in IMI waveguides ${ }^{28}$ GBSW losses are small even for very thin dielectric ridges because of the all-dielectric structure. This work opens disruptive perspectives in those sensing applications in which thin, patterned organic functional film are also able to provide field confinement on the chemically active area of the sensor.

Acknowledgment. This work is supported by the Piedmont Regional project CIPE 2008 "PHotonic biOsensors for Early caNcer diagnostICS (PHOENICS)", the Swiss National Science Foundation and the Science and Technology Atheneum Research Programme of the SAPIENZA University. Andreas Kern is acknowledged for his technical help in graphics handling.

\section{REFERENCES AND NOTES}

(1) Yeh, P.; Yariv, A. Optical surface waves in periodic layered media. Appl. Phys. Lett. 1978, 32, 104-105.

(2) Gaspar-Armenta, J.; Villa, F. Band-structure properties of onedimensional photonic crystals under the formalism of equivalent systems. J. Opt. Soc. Am. B 2004, 21, 405-412. 
(3) Shinn, M.; Robertson, W. M. Surface plasmon-like sensor based on surface electromagnetic waves in a photonic band-gap material. Sens. Actuators, B 2005, 105, 360-364.

(4) Guillermain, E.; Lysenko, V.; Orobtchouk, R.; Benyattou, T.; Roux, S.; Pillonnet, A.; Perriat, P. Bragg surface wave device based on porous silicon and its application for sensing. Appl. Phys. Lett. 2007, 90, 241116

(5) Descrovi, E.; Frascella, F.; Sciacca, B.; Geobaldo, F.; Dominici, L.; Michelotti, F. Coupling of surface waves in highly defined 1D porous silicon photonic crystals for gas sensing applications. Appl. Phys. Lett. 2007, 91, 241109

(6) Konopsky, V. N.; Alieva, E. V. Photonic crystal surface waves for optical biosensors. Anal. Chem. 2007, 79, 4729-4735.

(7) Robertson, W. M.; May, M. S. Surface electromagnetic wave excitation on one-dimensional photonic band-gap arrays. Appl. Phys. Lett. 1999, 74, 1800-1802.

(8) Ramirez-Duverger, A. S.; Gaspar-Armenta, J.; García-Llamas, R. Surface wave effect on light scattering from one-dimensional photonic crystals. Opt. Commun. 2007, 277, 302-309.

(9) Descrovi, E.; Sfez, T.; Dominici, L.; Nakagawa, W.; Michelotti, F.; Giorgis, F.; Herzig, H. P. Near-field imaging of Bloch surface waves on silicon nitride one-dimensional photonic crystals. Opt. Express 2008, 16, 5453-5464

(10) Descrovi, E.; Giorgis, F.; Dominici, L.; Michelotti, F. Experimental observation of optical bandgaps for surface electromagnetic waves in a periodically corrugated one-dimensional silicon nitride photonic crystal. Opt. Lett. 2008, 33, 243-345.

(11) Sfez, T.; Descrovi, E.; Dominici, L.; Nakagawa, W.; Michelotti, F.; Giorgis, F.; Herzig, H. P. Near-field analysis of surface electromagnetic waves in the bandgap region of a polymeric grating written on a one-dimensional photonic crystal. Appl. Phys. Lett. 2008, 93, No. 061108

(12) Liscidini, M.; Sipe, J. E. Analysis of Bloch-surface-wave assisted diffraction-based biosensors. J. Opt. Soc. Am. B 2009, 26, 279-289.

(13) Gizeli, E.; Lowe, C. R. Biomolecular Sensors; Taylor \& Francis: London, 2002

(14) Lal, S.; Link, S.; Halas, N. J. Nano-optics from sensing to waveguiding. Nat. Photonics 2007, 1, 641-648.

(15) Bozhevolnyi, S. I. Plasmonic nanoguides and circuits; Pan Stanford Publishing Pte Ltd: Singapore, 2008.

(16) Berini, P.; Charbonneau, R.; Lahoud, N. Long-range surface plasmons on ultrathin membranes. Nano Lett. 2007, 7, 1376-1380.

(17) Maier, S. A.; Pieter, G. K.; Atwater, H. A.; Meltzer, S.; Harel, E.; Koel, B. E.; Requicha, A. A. G. Local detection of electromagnetic energy transport below the diffraction limit in metal nanoparticle plasmon waveguides. Nat. Mater. 2003, 2, 229-232.

(18) Léveque, G.; Quidant, R. Channelling light along a chain of nearfield coupled gold nanoparticles near a metallic film. Opt. Express 2008, 16, 22029-22038.

(19) Akimov, A. V.; Mukherjee, A.; Yu, C. L.; Chang, D. E.; Zibrov, A. S.; Hemmer, P. R.; Park, H.; Lukin, M. D. Generation of single optical plasmons in metallic nanowires coupled to quantum dots. Nature 2007, 450, 402-406.

(20) Oulton, R. F.; Sorger, V. J.; Genov, D. A.; Pile, D. F. P.; Zhang, X. A hybrid plasmonic waveguide for subwavelength confinement and long-range propagation. Nat. Photonics 2008, 21, 496-500.

(21) Dintinger, J.; Martin, O. J. F. Channel and wedge plasmon modes of metallic V-grooves with finite metal thickness. Opt. Express $2009,17,2364-2374$

(22) Zia, R.; Selker, M. D.; Catrysse, P. B.; Brongersma, M. L. Geometries and materials for subwavelength plasmon modes. J. Opt. Soc. Am. A 2004, 21, 2442-2446.

(23) Dionne, J. A.; Sweatlock, L. A.; Atwater, A. C. Plasmon slot waveguides: towards chip-scale propagation with subwavelengthscale localization. Phys. Rev. B 2006, 73, No. 035407.

(24) Goto, T.; Katagiri, Y.; Fukuda, H.; Shinojima, H.; Nakano, Y.; Kobayashi, I.; Mitsuoka, Y. Propagation loss measurements for surface plasmon-polariton modes at metal waveguides on semiconductor substrates. Appl. Phys. Lett. 2004, 84, 852-854.

(25) Holmgaard, T.; Bozhevolnyi, S. I.; Markey, L.; Dereux, A. Dielectricloaded surface plasmon-polariton waveguides at telecommunication wavelengths: excitation and characterization. Phys. Rev. B 2008, 78, 165431

(26) Barrios, C. A.; Gylfason, K. B.; Sánchez, B.; Amadeu, G.; Sohlström, H.; Holgado, M.; Casquel, R. Slot-waveguide biochemical sensor. Opt. Lett. 2007, 32, 3080-3082.

(27) Quidant, R.; Weeber, J.-C.; Dereux, A.; Peyrade, D.; Chen, Y.; Girard, C. Near-field observation of evanescent light wave coupling in subwavelenght optical waveguides. Europhys. Lett. 2002, 57, 191-197

(28) Holmgaard, T.; Bozhevolnyi, S. I. Theoretical analysis of dielectricloaded surface plasmon-polariton waveguides. Phys. Rev. B 2007 , 75, 245405.

(29) Stefanon, I.; Blaize, S.; Bruyant, A.; Aubert, S.; Lerondel, G.; Bachelot, R.; Royer, P. Heterodyne detection of guided waves using a scattering-type Scanning Near-Field Optical Microscope. Opt. Express 2005, 13, 5553-5564. 